\title{
Editorial
}

Although GSP 6 (3) was meant to be a non-themed issue, now that the various contributions are finally published as a package it almost seems as if an "invisible" editorial hand has somehow managed spontaneously to produce a thematic issue after all, focusing on the impact of genomics on human health and existence.

Due to a common set of concerns, observations and ideas, these papers read as if they represent parts or voices in a coherent and orchestrated discussion. First of all, they unanimously point out how genomics, starting as a laboratory phenomenon, is now increasingly and emphatically becoming tangible and real, entering the life of citizens worldwide. Moreover, genomics is moving from general genomics to personalised genomics and personalised medicine. Genetic testing is spreading throughout society through options for direct-to-consumer testing and genomics is affecting parent-children relationships, having an impact on how we see and define ourselves, and on how concepts such as health and autonomy are framed and used.

These developments are enabled by a network of institutional settings, ranging from biobanks (such as the US-based eMERGE network) up to bioethical organisations (such as the Nuffield Council of Bioethics). Biobanks allow for genome-wide association studies that prepare the ground for a personalised medicine approach. Individuals are invited to assess their genomic profile through tests available on the internet, yielding information that they may apply not only to themselves but also to their offspring. And in the course of this process, ideas concerning human identity and diversity are becoming reframed.

For the ELSI community, all this entails an interesting challenge. It allows us to test and see whether the results of our exploratory and anticipatory research endeavours of the recent past will prove robust, feasible and thorough enough now that the brave new world of human genomics is finally coming into existence. In fact, this issue of GSP constitutes an effort in interdisciplinary dialogue between experts and stakeholders representing various perspectives, addressing a broad range of questions and concerns, ranging from issues raised by concrete genomics applications up to philosophical reflections on how genomics has affected our understandings and categorisations of nature and life. GSP 6 (3) provides a window into a future that has already begun.

\section{Hub Zwart}

Centre for Society and Genomics, Radboud University Nijmegen, the Netherlands 